\title{
Leonardo's vision of flow visualization
}

\author{
M. Gharib, D. Kremers, M.M. Koochesfahani, M. Kemp
}

\begin{abstract}
Leonardo's studies of cardiovascular systems, in more than 50 surviving pages from two phases of his research (around 1508-1509 and 1513), are a clear demonstration of his observational genius and progressive deduction of cardiac mechanics and the vascular system. He carried out a detailed hemodynamic study of the aortic valve motion and the role of the Sinus of Valsalva in the closure dynamics of the aortic valve, and he accurately correlated the formation of vortices with the separation of a retarded (shear) layer from the lips of the leaflets. In-vivo verification of vortex formation in the Sinus of Valsalva during the systolic phase awaited the application of modern phase-averaged magnetic resonance imaging techniques. Did Leonardo actually build the glass model he twice mentioned, thus performing the first scientific flow visualization of impulsive vortex formation or other fluid mechanical phenomena? Evidence in support of this possibility can be found in both the unusually schematic style he employed for this suite of drawings and the recent flow imaging results obtained in our laboratory through laserbased imaging techniques.
\end{abstract}

Received: 1 February 2002 / Accepted: 19 April 2002

Published online: 28 June 2002

(c) Springer-Verlag 2002

M. Gharib ( $\square)$

Graduate Aeronautical Laboratories and

Bioengineering Option,

California Institute of Technology,

Pasadena, CA 91125, USA

E-mail: mory@caltech.edu

Tel.: +1-626-3954453

D. Kremers

Division of Biology,

California Institute of Technology,

Pasadena, CA 91125, USA

M.M. Koochesfahani

Department of Mechanical Engineering,

Michigan State University,

Lansing, MI 48824, USA

M. Kemp

Department of the History of Art,

University of Oxford,

OX 11 PT Oxford, UK

The authors would like to take this opportunity to thank Dr. Mehrdad Zarandi for his contribution to DPIV experiments conducted for this study.

\section{Introduction}

Nowhere are we more tempted to look at a historical artist with modern eyes than with Leonardo da Vinci. He has often been seen as a universal genius, the prototype artistscientist-engineer of the Industrial Revolution. Freud claimed, "He was like a man who awoke too early in the darkness, while the others were all still asleep." To his 15th-century contemporaries, Leonardo was clearly a phenomenon - an artist who reformed his profession but completed few works, a designer of infinite inventiveness across the military and civil spheres, an impresario of courtly treats, an accomplished musician and an insatiable investigator of all visual phenomena - but no-one knew quite what to make of him. Though his Treatise on Painting, compiled by his heir, Francesco Melzi, and eventually published in 1651, was widely read for centuries, it was not until the late 19th century (Richter 1970), when his notebooks were extensively published for the first time, that his scientific and anatomical studies gained widespread exposure and acclaim.

Leonardo would not have recognized our contemporary opposition of art and science. In fact, our current uses of these words would puzzle him. In the unity of knowledge sought in the Middle Ages and the Renaissance, when Latin was still a living language, ars meant 'technique or practice', while scientia meant 'knowledge or theory', and Leonardo was certain no reliable model of nature could contain one without the other. He knew the power of visualization to be instrumental in depicting knowledge. He wrote that his representations "...will give true knowledge of their shapes, that neither the ancient writers nor the moderns would they ever have been able to give without an immense, tiresome and confused amount of writing and time". (Kemp 1972) And he goes on to say that "a very short way of drawing them (read visualizing them) from different aspects one gives a full and true knowledge of them" (Kemp 1989). He followed this approach while always trying to avoid "Sciences (read theories) that begin and end in the mind" (Kemp 1989).

His studies of cardiovascular systems, in more than 50 surviving pages from c. 1508-1509 and c. 1513 (O'Malley and Saunders 1983, Keele and Pedretti 1979), are a clear demonstration of his observational genius, theoretical intuitions, and progressive deduction of cardiac mechanics and the vascular system. The heart, a principal metaphor and mechanism of life, has presented an intellectual challenge throughout the history of mankind. Despite the intense curiosity shown by ancient philosophers and 
scientists, the role of the heart as a pumping muscle came late in the history of science. It is imperative to mention that most of the conjectures and beliefs regarding the nature of the cardiovascular system made by ancient Egyptians, Greeks, and Islamic physicians and philosophers were based on the application of a priori concepts to a modest base of anatomical knowledge. Dissection, particularly of human subjects, was uncommon, for a variety of social and religious reasons. It was only in the 13th century that dissection became more widely practiced in the education of doctors and as a form of public display (Carlino 1999). However, the purpose of these dissections was mainly to compliment the reading of standard anatomical texts rather than to effect radical discoveries and understand anew the physiology of the bodily systems.

\section{2}

\section{Leonardo's studies of the cardiovascular system}

Leonardo's depiction of coronary arteries in his later anatomical drawings can be considered a textbook example of accuracy in observation. He was the first to study the branching and size distribution of each arterial bifurcation, pointing out the constantly diminishing size of the blood vessels until they become invisible to the naked eye. During the course of his study of the cardiovascular system, he came to realize that the heart had four chambers, depicting them in his notebooks with lifelike detail. He was clearly fascinated by the mechanics of the heart.

Historically, Leonardo was the first to study in detail the small valves (tricuspid and mitral), actually identifying them as one-way mechanical regulating valves (Kemp 1972). Leonardo believed that blood surged in pulses from both ventricles of the heart, expending its energy in a process of ebb and flow as it moved inside the arteries towards other organs. Valves and vessels regulated and controlled the blood movement in its anatomical journey.
He carried out a detailed hemodynamic study of the aortic valve motion and the role of the Sinus of Valsalva in the closure dynamics of the aortic valve. In a series of drawings in the Royal Collection at Windsor and in other manuscripts (O'Malley and Saunders 1983, Keele and Pedretti 1979) he explained that when the heart contracts, blood from the lower ventricle (the left ventricle) rushes with 'impetus' through the cups of the aortic valve, then forms a vortex that grows and expends its energy by beating against the walls of the sinus and valve leaflets.

In a remarkable piece of writing, Leonardo described the formation of vortices as if he were observing them in action (Keele and Pedretti 1979). He wrote "... the middle of the blood (stream) which surges through the triangle $a b$ $c$ (aortic aperture, Fig. 1a) surges to a much greater height than that which surges along the sides of this triangle. This occurs so that the blood in the middle of the triangle directs its impetus straight upwards and that which surges along the sides distributes its impetus by lateral motion, and percusses against the front of the arches of the hemicycles (aortic sinus), and follows the concavity of this hemicycle, constantly passing downwards, until it percusses against the concavity at the base of this hemicycle (i.e. concavity of the cusps) and then by reflected motion turns upward and continues to revolve upon itself with a circular motion until it expands its impetus..."

In these writings, we note that Leonardo realized that solid surfaces retard the flow along the valve leaflet, which is perhaps the first documented observation of the effect of no-slip condition. He had already studied this phenomenon in water expelled from pipes, noting that the water "surges higher which is further from the walls of the pipe" (Keele and Pedretti 1979). The second interesting point is that he clearly understood that the impulsive motion along a wall would generate a layer that could possess eddying motion. He had extensively investigated such flow
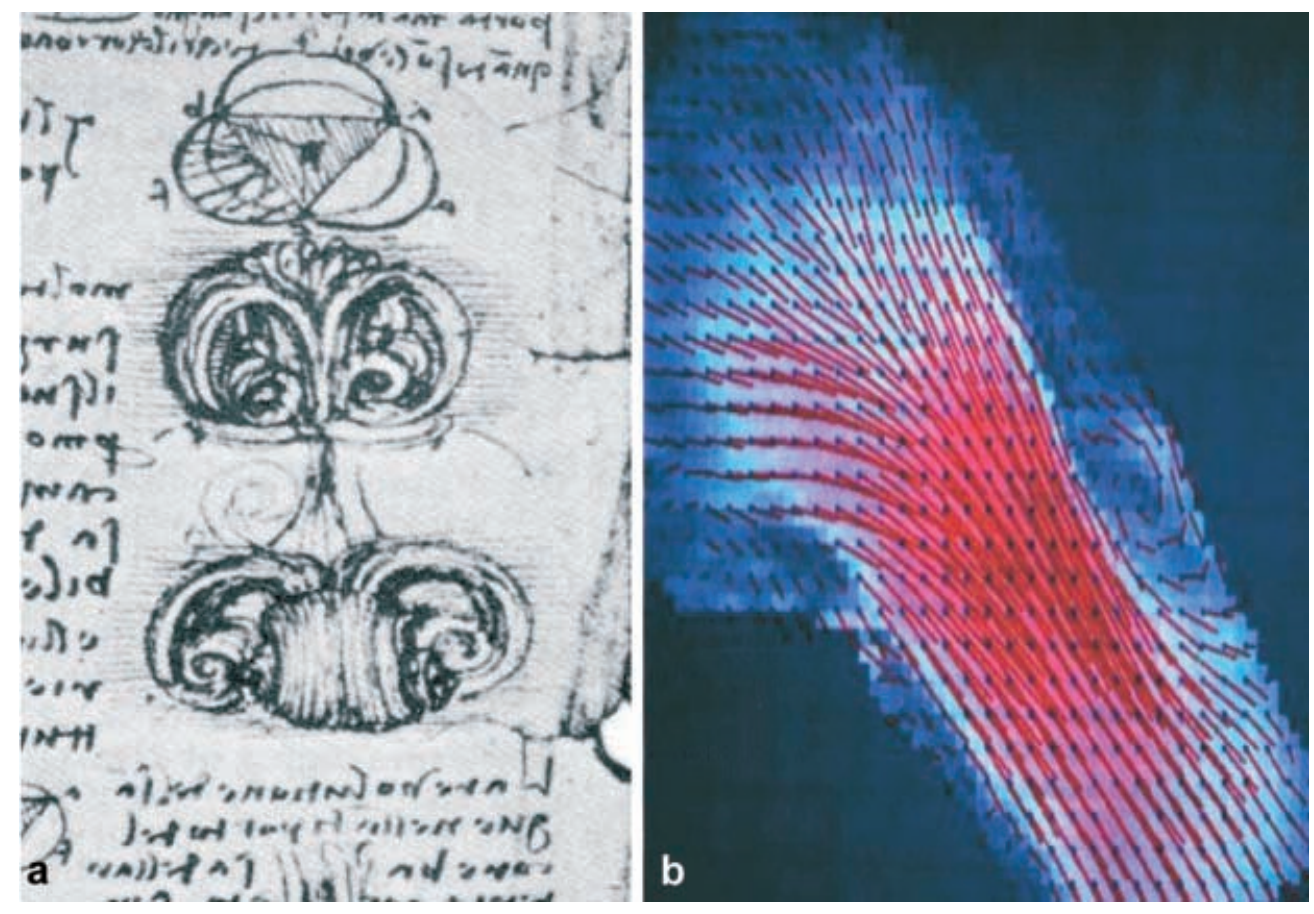

Fig. 1. a Leonardo da Vinci's depiction of aortic valve vortices in the left heart. (Detail of RL19116 recto - Courtesy of The Royal Collection (C) 2002, Her Majesty Queen Elizabeth II). b Velocity vectors from MRI in the left ventricular outflow tract, again showing vortices in the Sinus of Valsalva. (Courtesy of Dr. Tal Geva of Children's Hospital Boston) 
phenomena, especially in rivers and canals (Macagno 1988). In the same section that he described vortex formation in the Sinus of Valsalva, he accurately correlated the formation of vortices with the separation of a retarded (shear) layer from the lips of the leaflets. But perhaps the most important finding in his observations is the identification of vortex formation in the sinus as a mechanism for the valve's closure. In an attempt to find a function to fit a known form, he described vortex formation as being in perfect harmony with the aortic sinus and its curved geometry in providing a closure mechanism for the aortic valve (Keele and Pedretti 1979). A modern verification of this mechanism can be found in the work of Lee and Talbot (1979) who used flow visualization in a glass model to show a similar dynamic to those Leonardo described in his writings.

In-vivo verification of vortex formation in the Sinus of Valsalva during the systolic phase had to await the application of phase-averaged magnetic resonance imaging (MRI) techniques in mapping the velocity field inside the Sinus of Valsalva. Figure 1b (Tal Geva, Children's Hospital Boston, private communication) shows the velocity vector field of a systolic jet through the aortic valve and depicts the formation of the vortex behind one of the valve leaflets. In this image, obtained by MRI, the valve itself is not resolved. The reader can find more examples of MRI application in cardiovascular flow imaging in Kilner et al. (2000) and Yang et al. (1998).

One should feel perplexed by Leonardo's insight and the methods that he implemented to make his observations; for how could he observe flow around an aortic valve through opaque blood streams in a live subject, such as an ox or human? In this respect, we wondered if Leonardo had used an in-vitro approach to visualize the flow pattern around the artificial aortic valve and inside the Sinus of Valsalva.

A clue to this puzzle can be found in the form of a passage and sketch sheet at Windsor (Keele and Pedretti 1979) which is part of his detailed exploration of the vortex formation in the Sinus of Valsalva. On this folio he writes, "A mould of gypsum to be inflated inside with thin glass and then break it from head and foot. But first pour the wax into the gate of a bull's heart, that you may see the true form of this gate" (O'Malley and Saunders 1983,

Kemp 1989). On the same page (Fig. 2b), he demonstrated the design for the construction of an artificial heart valve; an innovation by itself that waited 500 years to be emu-

lated. Incidentally, we know that he used an injection technique to make a wax cast of the ventricles of an ox brain (Keele and Pedretti 1979).

In his earlier series of studies, around 1508-1509, (Keele and Pedretti 1979), Leonardo wrote, "make a proof [or 'test'] in glass and move inside it water and panic grass seed." According to Kemp (1972), "Thrilled by what he believed to be the complete solution of the formal and dynamic problems posed by this micro-part of the microcosm, he plans to construct a model, which would conclusively demonstrate the validity of the principles behind his solution. The model would be a compositive demonstration of effects from causes, in the manner of his early work; but, in line with his late methodology, the compositive phase is now rigorously preceded by the more inductive phase of accounting for observed forms in the context of causes." O'Malley and Saunders (1983) doubt that he ever used the model to prove his theory.

Given that historians have disagreed as to whether Leonardo actually performed any glass aorta experiments, let us examine some of his drawings more carefully.
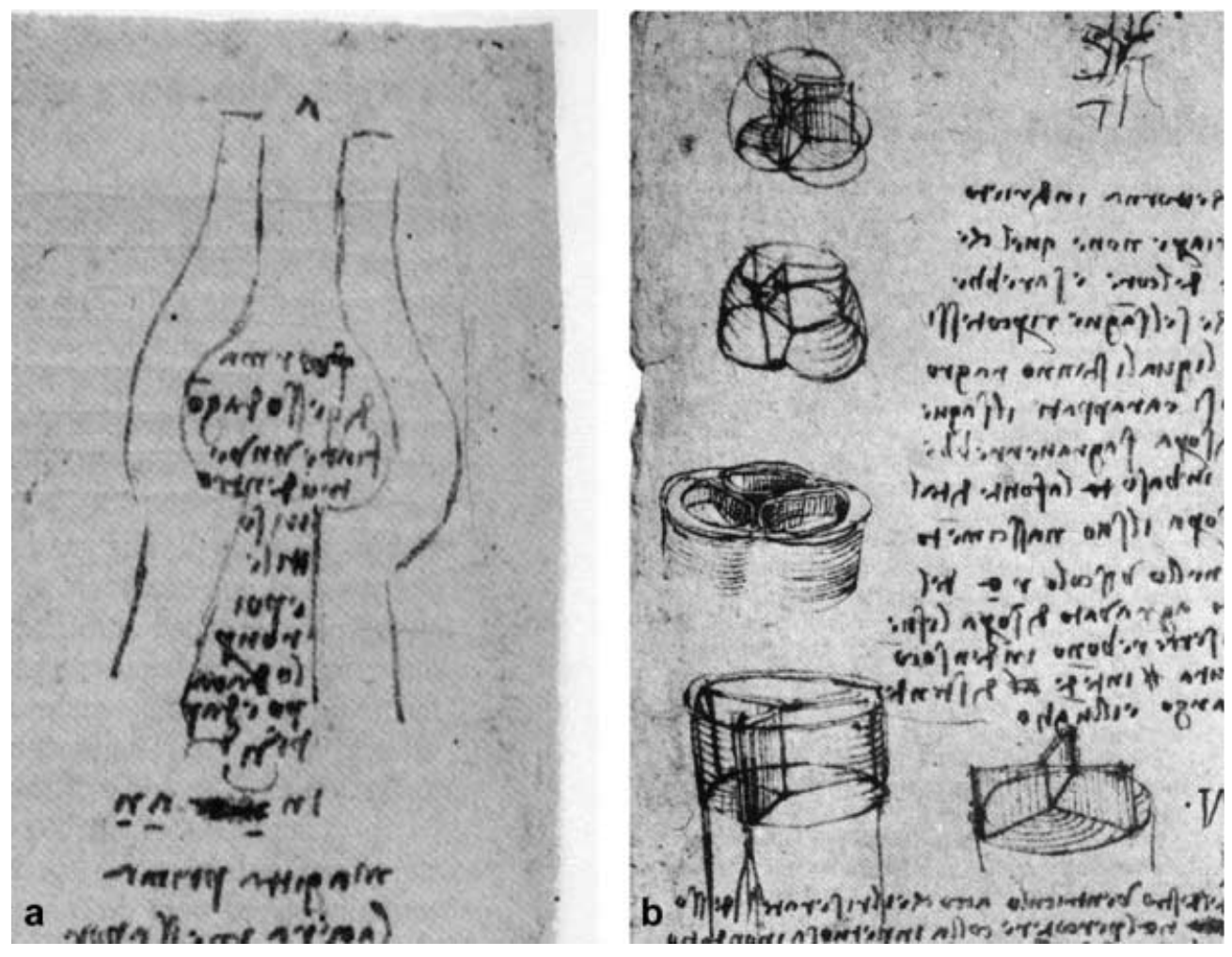

Fig. 2. a Schematic of Leonardo da Vinci's glass model of leftheart aortic track. b Detailed schematics and instruction for constructing artificial heart valves for the glass model by Leonardo. (Detail of RL19082 recto - Courtesy of The Royal Collection (C) 2002, Her Majesty Queen Elizabeth II) 

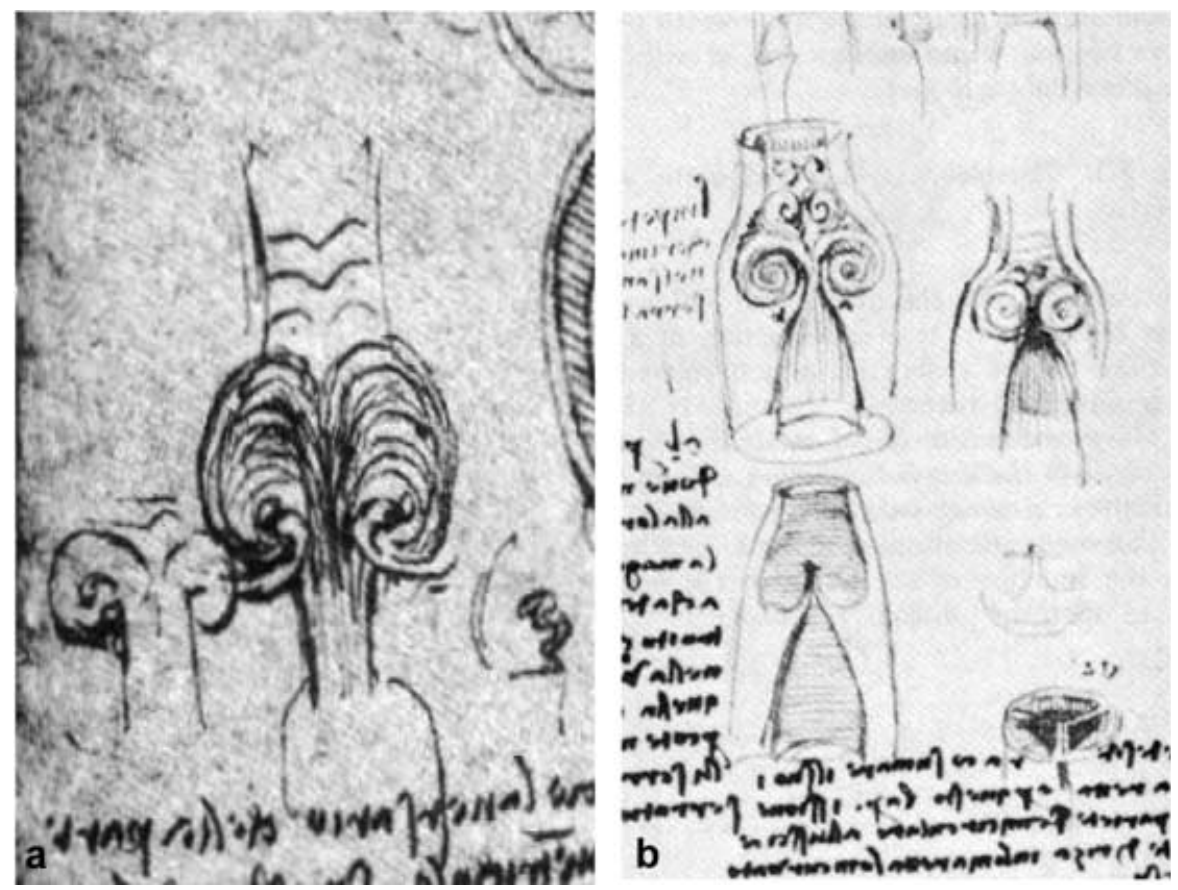

Fig. 3. Various schematics of flow in the aortic track by Leonardo. (Detail of RL19117 verso - Courtesy of The Royal Collection (C) 2002, Her Majesty Queen Elizabeth II)

Consider the schematics in Figs. 1a and 3. Common to all of these drawings is the simple shape of the figures, uncrowded by anatomical details. Now consider the way that Leonardo generally insisted in paying great attention to details and accuracy in his anatomical representations of the heart. He typically wrote "My depiction of the human body will be shown to you as if you had a real man before you ... as if you had the same member in your hand and went on turning it gradually until you had complete knowledge" (Kemp 1972). Even our present in-vivo MRI techniques cannot give as much detail as was routinely drawn by Leonardo.

Given his usual attention to anatomical details, one is forced to wonder about the schematic style of his sketches of the aortic root, valves, and Sinus of Valsalva drawn without the coronary out-tracks whenever he used them to depict the flow. Drawings for these sections show unrealistic stretching of the inlet and outlet tracks to and from the Sinus of Valsalva. His depictions (Keele and Pedretti 1979) of the flow track through the aortic valve are so simple that they resemble glass or plastic models of aortic root that are commercially available to modern researchers in biomedical laboratories. A significant number of the later studies show peculiar similarities to the geometrical sketch for the glass model rather than to an anatomically correct aortic orifice, (see Fig. 3). There is more research needed to clarify the precise sequence of events and the point at which data from the possible model appears to affect his visualization of the valve and its flow. For the moment, we can say that there are clear signs that the mode of his depictions strongly suggests that the model did indeed play a crucial role.

The evidence for glass model studies by Leonardo is reinforced by the recent flow imaging results obtained in Gharib's laboratory through laser-based imaging techniques, (see Fig. 4). A glass model of the aortic track is
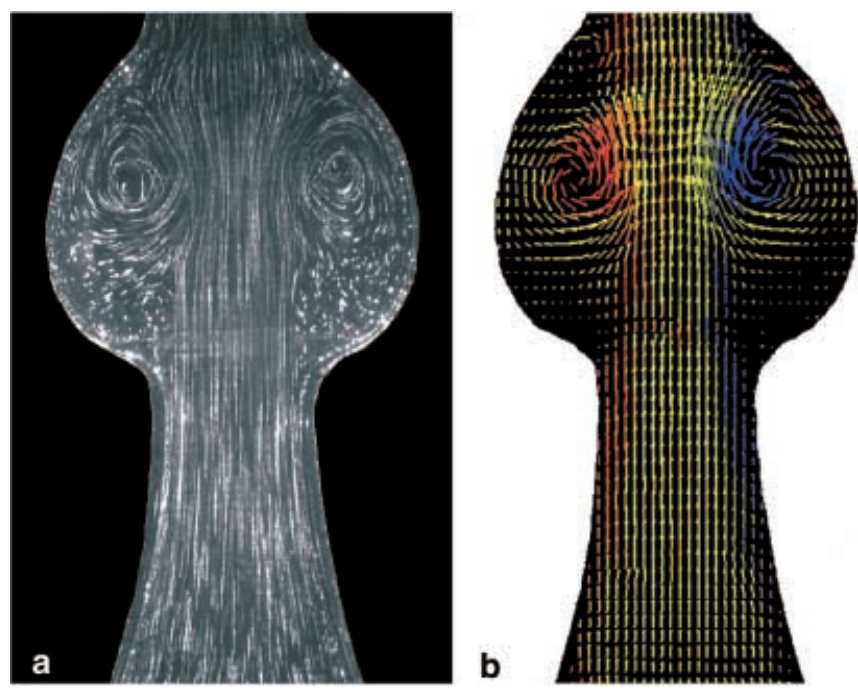

Fig. 4a, b. Flow in the glass model of the aortic track at two different instances during vortex formation. a particle streak visualization, b velocity map color-coded according to the vorticity level (blue: clockwise, red: counter-clockwise, yellow: zero vorticity)

constructed according to Leonardo's schematic in Fig. 2a. The flow enters from a reservoir, simulating the heart, and the pulsating flow signature of the heart is simulated using a pulsatile pump connected to the outlet of the glass model. Using laser sheet illumination, particle streak imaging reveals the qualitative flow patterns, whereas Doppler particle-imaging velocimetry (DPIV; Gharib and Dabiri 2000) allows the quantitative mapping of the velocity field. The images in Fig. 4 bear striking similarities to Leonardo's sketches of the flow, independently of the valve membranes, and attest to his observational power.

His studies on anatomy were based on his desire to analyze anatomy through mathematics, above all geome- 
try, revealing the invisible mechanics of force and power. In this respect, he used his flow visualization studies of aortic flow as a tool both for discovery and for validation, since both methods were exploited in a complimentary manner in science of the period. In keeping with an era in which procedures were not recorded in the modern manner, Leonardo himself did not indicate the sequence of events that resulted in his discoveries.

We also need to allow for the loss of his notes. To judge from the passages transcribed by the 16th-century editor of the Treatise on Painting (of which only a fraction can be traced in existing manuscripts), we can estimate that only about one-fifth of the artist's papers survive. This is explained in part by the editorial neglect and in part violence of men like Pompeo Leoni, a 16th-century sculptor at the Spanish court, who dismembered Leonardo's manuscripts. Leoni separated out the technical drawings in his possession from the 'artistic' ones, reassembling and selling the former as the Codex Atlanticus and the latter as a collection of folios at Windsor. It is likely that pages of technical studies, like those of the heart valves, were less likely to be prized by collectors than those connected with works of art and his more naturalistic studies of nature.

We also need to realize that he was not preparing his notes for publication in a scientific journal. There was no culture of systematic confirmation of experiments by one's peers. The order in which he recorded observations in his notebooks, as far as their chronology can be reconstructed, should be considered as the best evidence to prove the chronology of his hypothesis formation and experimental procedures.

Finally, we need to remember that with all of his experimental genius, he was obsessed by the mathematical structure of things. We must understand that 'mathematical' to Leonardo meant a search for the idealized geometries of antiquity, not our modern modelling of reality by equations as pioneered by Descartes and others in the 17th century.

\section{3}

\section{Concluding remarks}

Leonardo had a lifelong obsession with the fluid motion of water and air, and was fascinated by vortices. We propose it is conceivable that in pursuing his scientific methodology, Leonardo may have developed his physical understanding of the hemodynamics of the aortic valve purely based on his in-vitro flow visualization studies. In this case, his flow observations in the glass heart were, like his theories on the flow of rivers, more deductive than observed. He demonstrated this direction by interjecting his deductions of the physical laws of nature, including medieval impetus mechanics, into his almost real-time observations (Kemp 1989). For example, he wrote, "It is impossible for any part of blood to remain in the right upper ventricle (auricle) when its lower ventricle dilates, and this is proved thus. It has been stated that Nature always accomplishes her effect in the easiest way and in the shortest time possible" (Keele and Pedretti 1979).

Interestingly, the discovery of the circulatory system and identification of the heart as a driving pump by William Harvey (DeBakey and Gotto 1997), like Leonardo, depended on natural flow visualization, which he used to reach his important discovery of blood circulation. By observing the heart action of tiny live shrimp through their transparent body walls, he began to doubt the Galanic model. Harvey also made observations of a chick's heart still in its egg, where the naked eye can see the respective appearance and disappearance of tiny blood spots during the diastolic and systolic phases of the cardiac cycle.

In this respect, Leonardo believed in flow visualization as an important tool for discovery and exploration, and he masterfully followed this principle in his studies of heart valves. We believe there is strong evidence to suggest that Leonardo used his simple glass model to simulate flow dynamics inside the aortic root, he conducted the first scientific flow visualization in the history of science about 400 years ahead of Osborn Reynolds' celebrated pipe flow visualization studies.

\section{References}

Carlino A (1999) Books of the body. University of Chicago Press, Chicago

DeBakey ME, Gotto AM (1997) The new living heart. Adams Media Corporation, Holbrook, Mass.

Gharib M, Dabiri D (2000) Digital particle image velocimetry in flow visualization: techniques and examples. Imperial College Press, London

Kemp M (1972) Dissection and divinity in Leonardo's late anatomy. J Warbug Courtauld Inst 35:200-225

Kemp M (1989) Leonardo da Vinci: The marvelous works of nature and man. Dent \& Son, London

Keele K, Pedretti C (1979) Leonardo da Vinci. Corpus of anatomical studies in the collection of her majesty the queen at Windsor Castle. Harcourt Brace Jovanovich, New York

Kilner PJ, et al (2000) Asymmetric redirection of flow through the heart. Nature 404:759-761

Lee CSF, Talbot L (1979) A fluid-mechanical study of the closure of heart valves. J Fluid Mech 91:41-63

Macagno E (1988) Leonardian fluid mechanics. [a series of volumes progressively analyzing all Leonardo's water researches] IIHR Monographs, Iowa

O'Malley CD, Saunder JB (1983) Leonardo on the human body. Dover Publications, Mineola, N.Y.

Richter JP (1970) The notebooks of Leonardo da Vinci, Vols I \& II (Modern Edition). Dover Publications, Mineola, N.Y.

Yang GZ, Mohiaddin RH, Kilner PJ, Firmin DN (1998) Vortical flow feature recognition: a topological study of in-vivo flow patterns using MR velocity mapping. J Computer Assisted Topogr 22:577586 